\title{
Feeding the monsters at low frequencies: LOFAR \& the evolution of radio-loud AGN
}

\author{
Wendy L. Williams ${ }^{1 *}$, Huub J. A. Röttgering ${ }^{2}$, Reinout van Weeren ${ }^{3}$, Gabriela \\ Calistro Rivera ${ }^{2}$ \\ ${ }^{1}$ School of Physics, Astronomy and Mathematics, University of Hertfordshire, College Lane, \\ Hatfield AL10 9AB, UK \\ ${ }^{2}$ Leiden Observatory, Leiden University, P.O. Box 9513, 2300 RA Leiden, The Netherlands \\ ${ }^{3}$ Harvard-Smithsonian Center for Astrophysics, 60 Garden Street, Cambridge, MA 02138, USA \\ E-mail: w.williams5@herts.ac.uk
}

\begin{abstract}
Radio observations provide a unique view of AGN. New low frequency observations with LOFAR are allowing us to build statistically large samples at high redshifts to study the evolution of the different accretion modes of radio-loud AGN. Here we present a new deep $\left(120 \mu \mathrm{Jy}_{\text {beam }}{ }^{-1}\right)$, high resolution $(5 \times 7$ arcsec $)$ image of the Boötes field at $150 \mathrm{MHz}$ with LOFAR. The LOFAR radio catalogue is combined with the optical-infrared data available in this 7 square degree field. We use SED fitting to determine the host galaxy properties, including stellar mass and rest frame colours. Additionally, we use SED fitting, including the FIR emission, to separate High Excitation and Low Excitation radio-loud AGN (HERGs and LERGs), and use this information to study the the properties of their host galaxies out to redshifts of about 2 .
\end{abstract}

EXTRA-RADSUR2015 (*)

20-23 October 2015

Bologna, Italy

(*) This conference has been organized with the support of the Ministry of Foreign Affairs and International Cooperation, Directorate General for the Country Promotion (Bilateral Grant Agreement ZA14GR02 - Mapping the Universe on the Pathway to SKA)

\footnotetext{
* Speaker.
} 


\section{Introduction}

The growth and evolution of black holes plays a significant role in the framework of galaxy evolution. It is important then to understand the fueling mechanisms of Active Galactic Nuclei (AGN) and their 'feedback' processes by which the central black holes can control or terminate star formation in their host galaxies (see e.g. Best et al., 2006; Croton et al., 2006; Cattaneo et al., 2009, and references therein). From studies of radio-loud AGN, which are identified by synchrotron emission from their jets, it has become clear that there are two classes of sources distinguished by their Eddington-scaled accretion rates (e.g. Best \& Heckman, 2012): the High and Low Excitation Radio Galaxies (HERGs and LERGs). For reviews on the dichotomoy of the radio AGN population see Heckman \& Best (2014) and McNamara \& Nulsen (2012).

HERGs are associated with radiatively efficient accretion via an accretion disc (e.g. Shakura \& Sunyaev, 1973). These sources fit the paradigm of classic optical 'quasars' (Silk \& Rees, 1998), radiating across the electromagnetic spectrum (e.g. Barthel, 1989; Antonucci, 1993). This 'cold mode' or 'radiative mode' is characterised by strong optical emission lines and so are refered to as 'high-excitation' sources. On the other hand, LERGs, first noted by their lack of emission lines (Hine \& Longair, 1979), are thought to occur when hot gas accretes directly onto the supermassive black hole in a radiatively inefficient manner (Hardcastle et al., 2007) through advection dominated accretion flows (ADAFs, e.g. Narayan \& Yi, 1995). They lack any evidence of mid-infrared emission from dusty tori (Whysong \& Antonucci, 2004; Ogle et al., 2006) and accretion-related X-ray emission (Hardcastle et al., 2006; Evans et al., 2006). This 'radio mode', 'hot mode' or 'jet mode' in particular provides a direct feedback connection between the AGN and its hot gas fuel supply in the manner of work done by the expanding radio lobes on the hot intra-cluster gas. To understand how AGN and galaxies co-evolve we need to understand the cosmic evolution of radio sources in detail. Within the local universe $(z<0.3)$, the LERG/HERG populations are well understood: the fraction of galaxies which host a radio source, i.e. the radio-loud fraction, is a very steep function of host galaxy stellar mass (Best et al., 2005; Janssen et al., 2012), the radio-loud AGN population is dominated by LERGs (Best et al., 2006), the fraction of radio-loud AGN for the two classes have different realtionships with radio luminosity, galaxy color and star formation rate. A key open question is how the radio loud fraction, and radio source duty cyce, depend on host galaxy masses and colours for HERGs and LERGs at higher redshifts.

The main distinguishing point between HERGs and LERGs is their optical spectra. Best et al. (2014) provide the largest sample that is spectroscopically classified into jet- and radiative mode AGN at intermediate redshift $(z<1)$. In order to pursue studies with large samples at higher redshift a method is needed that does not require spectroscopy. Mid-infrared (MIR) quasar-selection techniques (Stern et al., 2005) are insufficient as they do not select all high excitation sources. Neither does selecting on X-ray emission alone (e.g. Hickox et al., 2009). Using high power sources out to $z \approx 4$, all of which have optical spectroscopy, Gürkan et al. (2014) have recently suggested that there is a clear division between LERGs and HERGs in the mid-IR-radio plane and a simple cut in $22 \mu \mathrm{m}$ luminosity can separate the populations. We have attempted to classify a sample of radio-loud AGN as HERGs and LERGs based on fits to their broad-band spectral energy distributions (SEDs), and use this to study the radio-loud fraction for the two populations between $0.5 \leq z<2$. 


\section{LOFAR observations of the Boötes field}

The LOw Frequency ARray (LOFAR) is a new generation radio telescope operating at $10-240 \mathrm{MHz}$ (van Haarlem et al., 2013). Its large instantaneous field-of-view, combined with multi-beaming capabilities, high-spatial resolution, and large fractional bandwidth make LOFAR an ideal instrument for carrying out large surveys of the sky which will enable more detailed studies of AGN at higher redshifts. We have made the first wide area $\left(19 \mathrm{deg}^{2}\right)$, deep $\left(\approx 120-150 \mu \mathrm{Jy}_{\text {beam }}{ }^{-1}\right)$, high resolution $(5.6 \times 7.4 \mathrm{arcsec})$ LOFAR High Band Antenna image of the Boötes field made at $130-169 \mathrm{MHz}$. This image is at least an order of magnitude deeper and 3-5 times higher in angular resolution than previously achieved for this field at low frequencies. Advanced calibration and processing techniques are needed to obtain such deep high-fidelity images at low radio frequencies. In particular, direction-dependent effects (DDEs) caused by the ionosphere and station beam shapes need to be corrected for. To do this, we have used the facet calibration strategy, introduced by (van Weeren et al., 2016). The final image is shown in Fig. 1. The central rms noise level is relatively smooth and $\sim 125 \mu \mathrm{Jy}_{\text {beam }}{ }^{-1}$, and 50 per cent of the map is at a noise level below $180 \mu \mathrm{Jy}_{\text {beam }}{ }^{-1}$. The final radio source catalogue consists of 5652 sources with flux densities between $0.4 \mathrm{mJy}$ and $5 \mathrm{Jy}$ over an area of $19 \mathrm{deg}^{2}$.

\section{Radio AGN in the Boötes field}

The Boötes field is one of the largest of the well-characterised extragalactic deep fields and was originally targeted as part of the NOAO Deep Wide Field Survey (NDWFS; Jannuzi et al., 1999) covering $\approx 9 \mathrm{deg}^{2}$ in the optical and NIR. There is a wealth of ancillary data available for this field, including X-ray, UV, MIR and FIR. For the sample of 889,007 optical sources in the Brown et al. (2007) psf-matched photometry catalogue with $m_{I} \leq 24$ mag, we have performed spectral energy distribution (SED) fitting to determine photometric redshifts and galaxy parameters, including stellar mass, star formation rates and rest-frame colours.

The LOFAR radio sources are matched with optical galaxies using the likelihood ratio (LR) method (Richter, 1975) to quantify the probability of an I-band optical source being the true host of a given radio source. We also inspected the images of each of the radio sources overlaid upon the corresponding optical images, to classify their radio morphologies.

\subsection{HERG/LERG separation}

We have attempted to separate the radio sources into HERGs and LERGs based on further SED fitting, now including the FIR fluxes, to determine the relative AGN and galaxy contribution to their IR emission. This fitting was done using the Markov chain Monte Carlo algorithm AGNFITTER (Calistro Rivera et al., 2015, in prep). The FIR fluxes provide an important constraint to separate the starformation and AGN components. The total active galaxy model in AGNFITTER consists of the superposition of the host galaxy emission and the nuclear AGN emission. The host galaxy is modelled as a combination of a stellar emission (GA) and the reprocessed emission of cold/warm dust in starburst (SB) regions. At nuclear scales, the AGN emission is modelled as a combination of an accretion disk component and a hot dust torus (TO) component. Some examples of the AGNFITTER SEDs with their components are shown in Fig. 2. 


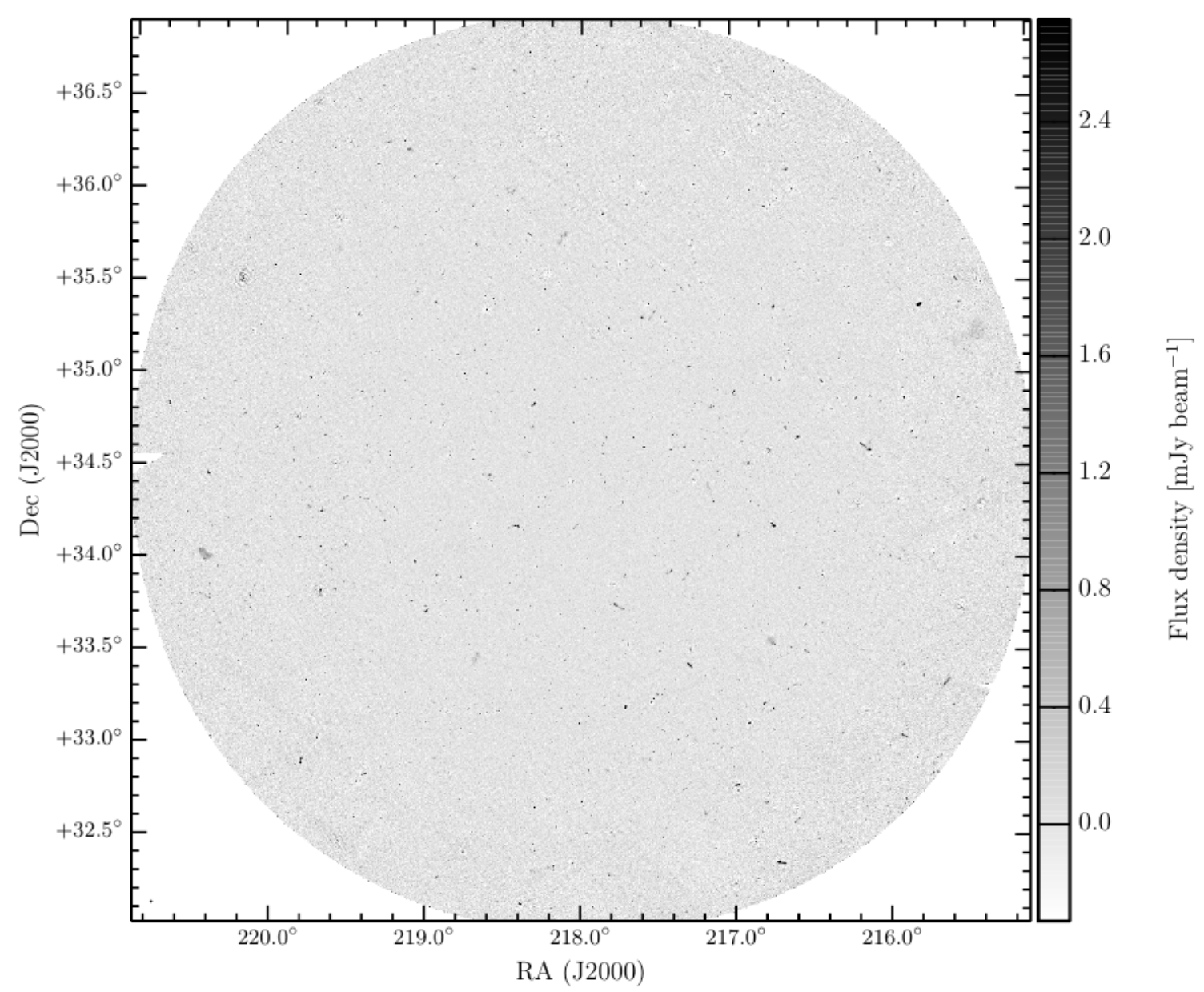

Figure 1: Greyscale map showing the LOFAR image, which covers $\approx 19 \mathrm{deg}^{2}$. The greyscale shows the flux density from $-3 \sigma_{\text {cen }}$ to $25 \sigma_{\text {cen }}$ where $\sigma_{\text {cen }}=125 \mu \mathrm{Jy} \mathrm{beam}^{-1}$ is the approximate rms in the mosaic centre.

Through this fitting we can disentangle the host galaxy and AGN luminosities that contribute to the IR emission. While the AGN contribution, which affects specifically the MIR regime, arises mainly from the torus luminosity $L_{T O}$, the contribution from galaxy emission takes into account both the stellar emission $L_{G A}$ and the reprocessed emission by cold/warm dust $L_{S B}$, since both have contributions in the MIR. For comparison, we compute luminosities integrated over the fitted templates across the wavelength range $1 \mu \mathrm{m}<\lambda<8 \mu \mathrm{m}$. From the AGNFITTER output we calculated the AGN torus fraction,

$$
f_{T O}=\frac{L_{T O}}{L_{T O}+L_{G A}}
$$

as a measure of the fraction of IR-emission that is coming from the torus with respect to that from the galaxy, independent of the SB component. We take a conservative boundary value of $f_{T O}=0.25$ and classify sources with $f_{T O}<0.25$ as LERGs and $f_{T O}>0.25$ as HERGs. Table 1, including those taken from the local reference sample, from which it can be seen that the fraction of HERGs increases between $0.5 \leq z<1.0$ and $1.0 \leq z<1.5$. The percentage of HERGs and 

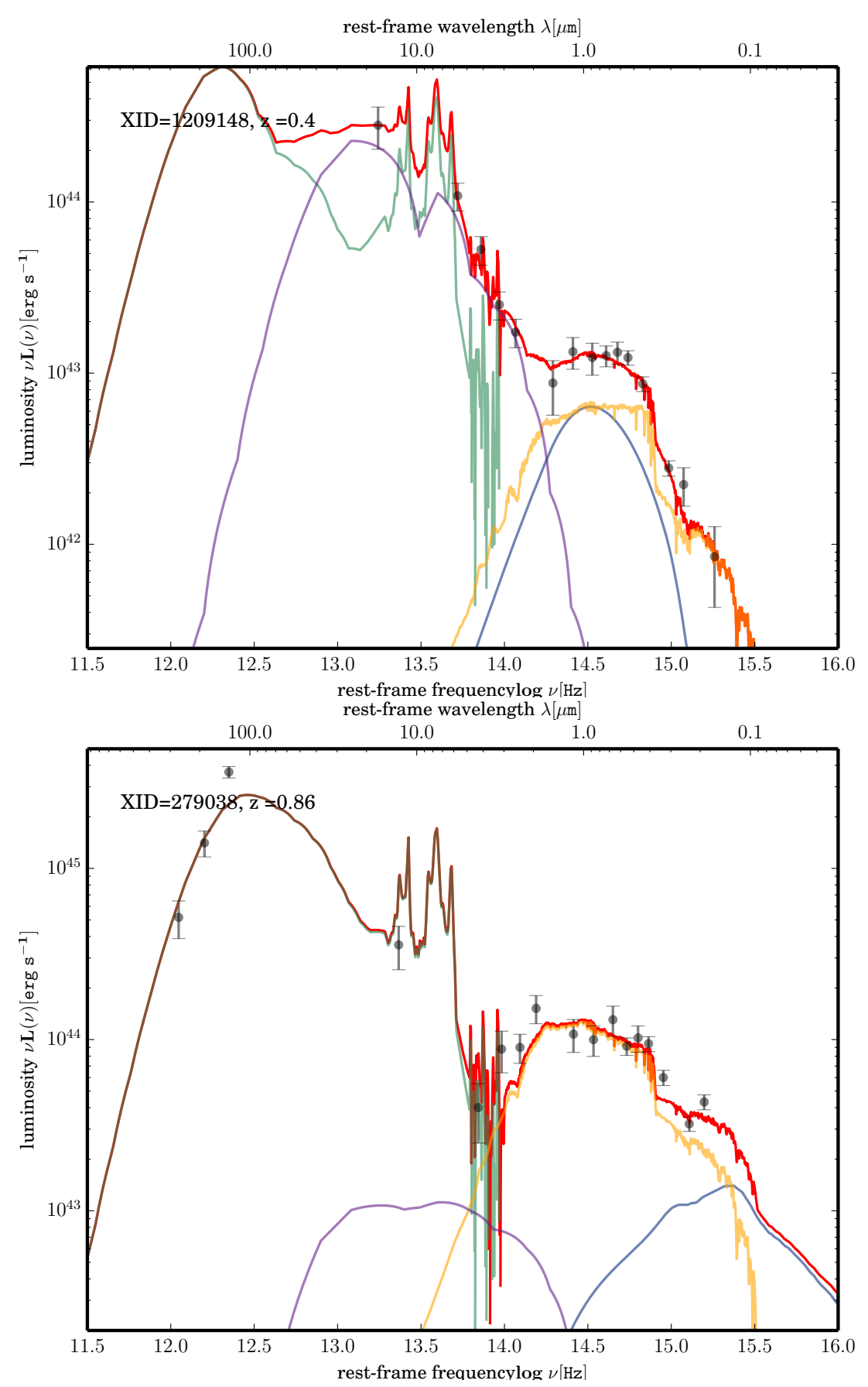

Figure 2: Examples of good quality SED Fits with significant star formation contribution (high $f_{S B}$ values) for top HERGs and bottom LERGs.The maximum likelihood realisation is plotted (red) and the fitted components are the AGN torus (purple), the starburst (green), the galaxy (yellow) and the blue bump (blue) with the total SED in red. 
Table 1: Number of sources classified as HERGs and LERGs in the three redshift intervals.

\begin{tabular}{llll}
\hline$z$ & $N$ & LERGs (\%) & HERGs ( \%) \\
\hline $0.01-0.3$ & 3736 & $3066(96 \%)$ & $121(4 \%)$ \\
\hline $0.5-1.0$ & 173 & $52(30 \%)$ & $121(69 \%)$ \\
$1.0-1.5$ & 384 & $72(18 \%)$ & $312(81 \%)$ \\
$1.5-2.0$ & 390 & $67(17 \%)$ & $321(82 \%)$ \\
\hline
\end{tabular}

Table 2: Number of sources classified as blue and red HERGs and LERGs in the three redshift intervals.

\begin{tabular}{llllll}
\hline$z$ & $N$ & BLERGs (\%) & RLERGs (\%) & BHERGs (\%) & RHERGs (\%) \\
\hline $0.01-0.3$ & 3736 & $148(3.9 \%)$ & $2918(78 \%)$ & $61(1.6 \%)$ & $60(1.6 \%)$ \\
\hline $0.5-1.0$ & 173 & $17(9 \%)$ & $32(18 \%)$ & $89(51 \%)$ & $28(16 \%)$ \\
$1.0-1.5$ & 384 & $52(13 \%)$ & $20(5 \%)$ & $248(64 \%)$ & $57(14 \%)$ \\
$1.5-2.0$ & 390 & $55(14 \%)$ & $12(3 \%)$ & $301(77 \%)$ & $10(2 \%)$ \\
\hline
\end{tabular}

LERGs within each redshift interval is given with respect to the total number of radio sources in that interval.

Having classified the sources as HERGs and LERGs based on their AGN contribution in the MIR, we study the distribution of the radio-loud AGN with respect to their optical host colours and masses (Fig. 3). We show for four redshift intervals, a plot of the ${ }^{0.1}(u-r)$ colour $^{1}$ versus stellar mass for optical sources and radio sources (except in the lowest redshift bin where the equivalent parameters are spectroscopic). The $f_{T O}$ values for the radio sources are shown in colour and the distributions of stellar mass and colour are shown for the optical sources, the radio sources and the HERGs and LERGs. In all redshift intervals it is clear that the distributions of optical and radio sources are different, as expected. In all cases the radio source distribution is skewed towards more massive galaxies and redder galaxies compared to the full galaxy population. The distributions of HERGs and LERGs are also different, in that the HERGs occur over a wider range of stellar masses $10^{9}<M_{*} / \mathrm{M}_{\odot}<10^{11.5}$ and over a wider range of colours. LERGs are much more likely to be in massive red galaxies.

The numbers of blue and red LERGs (BLERGs and RLERGs respectively) and HERGs (BHERGs and RLERGs respectively) within each redshift interval are listed in Table 2. This table also gives the fraction of each subgroup with respect to the number of radio-loud sources in each redshift interval.

\section{Summary}

We have constructed a catalogue of photometric redshifts, stellar masses, and rest-frame colours via SED fitting of the multi-band photometry catalogue (Brown et al., 2007) available for the Boötes field. We have identified optical counterparts from this catalogue of sources to radio sources from a

\footnotetext{
${ }^{1}$ This colour is determined from the ${ }^{0.1} u$ and ${ }^{0.1} r$ bands, defined as the AB magnitudes in the SDSS $u$ and $r$ bands at $z=0.1$.
} 

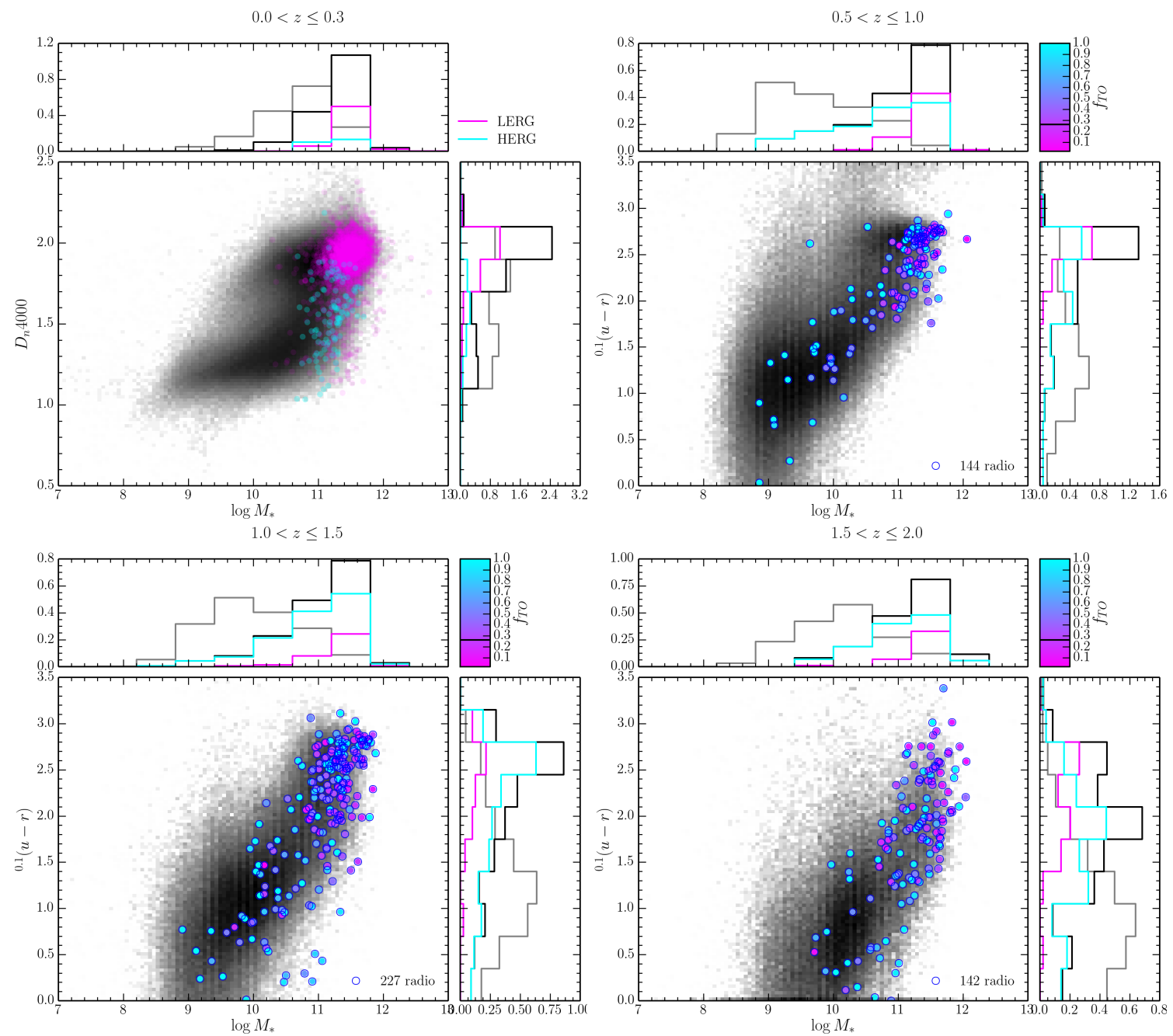

Figure 3: Colour, ${ }^{0.1}(u-r)$, versus stellar mass in the fours redshift intervals (in each panel). The density of optical sources in the Boötes field is plotted in black, in log units, and the radio sources are plotted with blue circles, with the fill-colour indicating their torus fraction, $f_{T O}$. The subpanels show the stellar mass (top) and colour distributions (right). The normalised distribution of optical sources is shown in grey, and that of all the radio sources in black. The HERGs and LERGs (separated at $f_{T O}=0.25$ ) are shown in cyan and magenta respectively, normalised to the total number of radio sources. The local sample, $0.01 \leq z<0.3$, is taken from SDSS and includes spectroscopic parameters.

new deep $150 \mathrm{MHz}$ LOFAR image of the Boötes field. For a well-defined sub-sample of 974 radio sources with optical matches between $0.5 \leq z<2$ and radio-powers above $P_{150 \mathrm{MHz}}>10^{25} \mathrm{~W} \mathrm{~Hz}^{-1}$, we have done additional SED fitting, including the FIR emission, to determine the relative contribution of AGN and galaxy emission in the MIR. From these results we have defined populations of HERGs and LERGs and studied the radio-loud fraction as a function of galaxy mass and colour. In summary, we have found the following:

1. The fraction of HERGs increases between $z=0.5$ and $z=2$; 
2. While most LERGs are found in 'red and dead' (non-star forming) galaxies, at $z>1$ there is a population of LERGs in star forming galaxies; and HERGs are found in both star forming and dead galaxies;

3. LERGs are predominantly found in more massive galaxies than HERGs;

4. LERGs are predominantly found in redder galaxies, but at higher redshifts are bluer;

5. The radio-loud fraction for blue HERGs increases with redshift while that for red HERGs decreases with redshift for all masses

\section{References}

Antonucci R., 1993, ARA\&A, 31, 473

Barthel P. D., 1989, ApJ, 336, 606

Best P. N., Heckman T. M., 2012, MNRAS, 421, 1569

Best P. N., Kaiser C. R., Heckman T. M., Kauffmann G., 2006, MNRAS, 368, L67

Best P. N., Kauffmann G., Heckman T. M., others 2005, MNRAS, 362, 25

Best P. N., Ker L. M., Simpson C., Rigby E. E., Sabater J., 2014, MNRAS, 445, 955

Brown M. J. I., Dey A., Jannuzi B. T., others 2007, ApJ, 654, 858

Calistro Rivera G., Lusso E., Hennawi J. H., Hogg D. W., 2015

Cattaneo A., Faber S. M., Binney J., others 2009, Nature, 460, 213

Croton D. J., Springel V., White S. D. M., others 2006, MNRAS, 365, 11

Evans D. A., Worrall D. M., Hardcastle M. J., Kraft R. P., Birkinshaw M., 2006, ApJ, 642, 96

Gürkan G., Hardcastle M. J., Jarvis M. J., 2014, MNRAS, 438, 1149

Hardcastle M. J., Evans D. A., Croston J. H., 2006, MNRAS, 370, 1893

Hardcastle M. J., Evans D. A., Croston J. H., 2007, MNRAS, 376, 1849

Heckman T. M., Best P. N., 2014, ARA\&A, 52, 589

Hickox R. C., Jones C., Forman W. R., others 2009, ApJ, 696, 891

Hine R. G., Longair M. S., 1979, MNRAS, 188, 111

Jannuzi B. T., Dey A., NDWFS Team 1999 Vol. 31 of BAAS. p. 1392

Janssen R. M. J., Röttgering H. J. A., Best P. N., Brinchmann J., 2012, A\&A, 541, A62

McNamara B. R., Nulsen P. E. J., 2012, New Journal of Physics, 14, 055023

Narayan R., Yi I., 1995, ApJ, 452, 710

Ogle P., Whysong D., Antonucci R., 2006, ApJ, 647, 161

Richter G. A., 1975, Astronomische Nachrichten, 296, 65

Shakura N. I., Sunyaev R. A., 1973, A\&A, 24, 337

Silk J., Rees M. J., 1998, A\&A, 331, L1

Stern D., Eisenhardt P., Gorjian V., others 2005, ApJ, 631, 163

van Haarlem M. P., Wise M. W., Gunst A. W., others 2013, A\&A, 556, A2

van Weeren R. J., Williams W. L., Hardcastle M. J., others 2016, ArXiv e-prints

Whysong D., Antonucci R., 2004, ApJ, 602, 116 